\title{
The Effect of Perioperative Psychological Intervention on the Surgery Patient Nursing Effect Score
}

\author{
Li Zhang ${ }^{1^{*}}$ and Deting $\mathrm{He}^{2}$ \\ ${ }^{1}$ Operation Room, Huoqiu Hospital of Traditional Chinese Medicine, Huoqiu, Anhui 237400, China \\ ${ }^{2}$ Department of Anesthesia, Huoqiu Hospital of Traditional Chinese Medicine, Huoqiu, Anhui 237400, China
}

\begin{abstract}
Objective: Discussed the effect of perioperative comprehensive psychological intervention on the surgery patient nursing effect score. Method: 80 cases undergoing elective general anesthesia cholecystectomy, ASA I or II, all genders, age 26-45 and weight 47-78 kg were selected. All patients were divided into two groups at random: the control group and the intervention group. All of the patients in two groups had no communication disorders and mental illness. The control group received conventional perioperative interview, in the intervention group, in addition to the conventional perioperative interview, the patients also received general psychological support and guidance, and emotions loose training and method of demonstration. Self-rating anxiety scale scores before and after psychological intervention, the SD and HR in the morning of surgery as well as the surgery patient nursing effect score after surgery were evaluated. Results: There was no statistical significance in gender, age, degree of education, occupation and operation method between two groups $(p>0.05)$. The difference of self-rating anxiety scale scores between two groups before intervention was not statistical significant $(p>0.05)$; the difference of self-rating anxiety scale scores between the intervention group three days after surgery and the control group was statistical significant $(p<0.05)$. The difference of surgery patient nursing effect score after surgery between the intervention group and the control group was statistical significant $(p<0.05)$; Conclusion: Perioperative psychological intervention could improve the sleep, relieve the anxiety emotion of patients, lower the SAS scores and increase the surgery patient nursing effect score. It also could speed up the recovery and the acceptance of biologypsychology-social medicine model, increase the satisfaction for treatment.
\end{abstract}

\section{KEYWORDS}

Perioperative period

Psychological intervention

Nursing effect score

\section{Introduction}

The psychological intervention is that apply the strategic impact to the psychological health problem and behavior of individuals and groups under the guidance of psychological theory, in order to achieve the change toward to expected target. The follow study was performed in order to

Copyright (c) 2012 Li Zhang and Deting He

doi: 10.18686/jn.vli1.4

Received: July 20, 2012; Accepted: September 18, 2012; Published online: November 25, 2012

This is an open-access article distributed under the terms of the Creative Commons Attribution Unported License (http://creativecommons.org/ licenses/by-nc/4.0/), which permits unrestricted use, distribution, and reproduction in any medium, provided the original work is properly cited.

${ }^{*}$ Corresponding author: Operation Room, Huoqiu Hospital of Traditional Chinese Medicine, Huoqiu, Anhui 237400, China. E-mail: lili_ zh882@sina.com determinate the effect of psychological intervention to the self-rating anxiety scale score of perioperative patients and surgery patient nursing effect score after surgery.

\section{Materials and methods}

80 cases undergoing elective general anesthesia cholecystectomy, ASA I or II, all genders, age $26 \sim 60$, weight $47 \sim 78 \mathrm{~kg}$ were selected. All patients were divided into two groups at random: the control group and the intervention group. There was no statistical significance in gender, age, degree of education, occupation and operation method between two groups $(p>0.05)$. All of the patients in two groups had no communication disorders and mental illness, all of them didn't use the analgesic pump. The control group received conventional perioperative interview, in the intervention group, in addition to the conventional perioperative interview, the patients also received general 
psychological support and guidance, as well as psychological intervention by emotions loose training and method of demonstration. Three days before surgery, the anesthesiologist entered the room to visit, communicated with patients, and distributed the self-Rating Anxiety Scale (SAS) to patients in order to score [1]. The scale included 20 items (level 1-4), the items of 5, 9, 13, 17 and 19 were reversely calculated, the total scores of 20 items multiplied by 1.25 , rounded to integer, that was the standard score. Before self-assessment, the patients should understand the filling method of the whole scale and the meaning of each question. Then the patients performed self-assessment independently in the case of not influenced by others. In general, the assessment would complete in $10 \mathrm{~min}$. Lower than 50 scores was normal, 50-59 scores was mild anxiety, 60-69 scores was moderate anxiety and higher than 70 scores was serious anxiety. The main psychological reactions of the patients before surgery found by communication were anxiety of operation and sleep disorders. In short, the reasons for anxiety included: (1) The patients did not fully understand the operation safety, especially didn't understand the anesthesia, accounted for about 90\%. (2) Worried about the effects of surgery, they were not sure of success and fastidious about the medical workers. (3) $30 \%$ of patients were afraid of pain, but didn't want to use the analgesia pump. (4) Warried about the family relationships cost of treatment and the effect to the future work and study. For the above reasons, communicated more with the patients in the intervention group, established a good doctor-patient relationship and performed the psychological intervention. The methods of intervention included: (1) General psychological support and guidance: listen patiently to the complains of patients, solve the problem pertinently, provide the patients with the detailed course of anesthesia in time, acquaint the patients with real information. It will relieve the fear of patients, enhance their patience. Introduce the anesthetic method and the feeling during anesthesia and operation, inform the patients that the advanced anesthesia equipment and scientific technique of anesthesia can guarantee the patient's safety during operation and avoid post-operative pain. Some traumatic preoperative preparation, such as stomach tube and urethral catheter can perform after anesthesia, in order to reduce the adverse reaction, fear and repellency of surgical anesthesia; (2) The control technology of anxiety behavior: use the emotions loose training and method of demonstration. The timely application of those intervention methods can minimize the preoperative anxiety of patients, so that the patients smoothly through anesthesia and operation period, promote the rehabilitation of patients. The emotions loose training can effectively control anxiety by relaxation, deep breath and coughing exercises, is the simple and common method to relieve the preoperative anxiety and intraoperative pain. The abdominal breathing relaxation method applied in the study as follow: sitting or lying position, one hand places on the chest and the other on the abdomen, gradually slow down the breathing rate; Do deep breathing, until you could feel that the hand on the abdomen raise and the hand on the chest keep substantially immobile. Do natural breath after remaining a moment, subvocalize 1, 2, 3 and so on. When breathe, feel the fall back of the hand on the abdomen, feel the feeling of the natural relaxation of systemic muscle as well as comfort and tranquility. Do it for 10-20 minutes each time and 2-3 times per day. Request the patients to perform the above methods consciously during and after surgery [2]. The principle of the emotions loose training is that the anxiety of patients would result in polypnea and breath which based on the thoracic breathing, in turn, the thoracic breathing stimulate the chest vagus nerve, lead to higher and stronger anxiety and tension reaction. The circulation could be blocked by abdominal breathing, weaken the feeling of systemic tension, lower the degree of anxiety. The method of demonstration: the patients are arranged to the operating room in which the patient's age, gender and operative type are the same or similar, in order to learn how the patients with good effect to come over the anxiety. Encourage the patients to come over the fear by watching videos and the experiences of Successful surgery patients. In the evening before surgery, visit the patient again. Record the SD and HR of the patient before anesthesia, record the nursing effect score of the patients after surgery, on the third day after surgery, and record the self-rating anxiety scale scores again.

Create a database by Microsoft Excel, the statistical analysis is performed by SPSS 13.0 statistical software. All the measurement data is expressed as mean \pm standard deviation $(\bar{x} \pm s)$, the measurement data is test by the $t$ test and the enumeration data is teat by the $x^{2}$ test, when $p<0.05$, the difference is statistical significant.

\section{Results}

There was no statistical significance in age, gender, weight, degree of education, and the time of operation between two groups $(p>0.05)$. See Table 1 .

The patient's feeling of anxiety is obviously relieved after peroperative psychological intervention, the scores of nursing effect is increased significantly too, compared to the control group, the difference was statistically significant $(p<0.05)$. See Table 2.

There is no difference in the heart rate and blood pressure between two groups before the psychological nursing intervention. After the psychological intervention, compared to the test group, the control patient's heart rate increased and the blood pressure raised, the difference is statistically significant $(p<0.05)$. See Table 3 .

\section{Discussion}

Along with the formation of bio-psycho-social medicine model, the disease-centered concept change to the human 
Table 1. The comparison of general information of patients.

\begin{tabular}{cccccc} 
& Age & Weight & Gender & The degree of education & Operation time \\
\cline { 2 - 6 } & (years, $\bar{x} \pm \mathrm{s})$ & $(\mathrm{kg}, \overline{\mathrm{x}} \pm \mathrm{s})$ & (female/male ratio) & $\begin{array}{c}\text { (High school or above/ } \\
\text { high school or below) }\end{array}$ & $(\mathrm{min}, \overline{\mathrm{x}} \pm \mathrm{s})$ \\
\hline The intervention group & $35 \pm 4$ & $67 \pm 10$ & $20 / 20$ & $26 / 14$ & $74 \pm 12$ \\
The control group & $37 \pm 5$ & $69 \pm 7$ & $19 / 21$ & $27 / 13$ & $75 \pm 11$ \\
\hline
\end{tabular}

Notes: Compared to the control group, $p>0.05$.

Table 2. Self-rating anxiety scale scores and the nursing effect scores before and after psychological intervention.

\begin{tabular}{ccccc}
\hline Group & SAS before intervention & $\begin{array}{c}\text { SAS three days after } \\
\text { surgery }\end{array}$ & $\begin{array}{c}\text { Psychological nursing effect } \\
\text { score before intervention }\end{array}$ & $\begin{array}{c}\text { Psychological nursing effect } \\
\text { score three days after surgery }\end{array}$ \\
\hline The intervention group & $41.25 \pm 9.23$ & $42.59 \pm 9.53^{* *}$ & $8.57 \pm 1.34$ & $15.63 \pm 2.31^{* *}$ \\
The control group & $40.98 \pm 10.67$ & $49.83 \pm 10.65$ & $8.46 \pm 1.25$ & $8.56 \pm 1.36$ \\
\hline
\end{tabular}

Notes: Compared to the control group, ${ }^{* *} p<0.05$.

Table 3. Change of HR and SD between two groups before and after intervention.

\begin{tabular}{ccccc}
\hline Group & HR before intervention & HR in the morning of surgery & SD before intervention & SD in the morning of surgery \\
\hline Intervention group & $84.27 \pm 7.23$ & $84.93 \pm 7.68^{*}$ & $124.60 \pm 19.55$ & $126.43 \pm 18.65^{*}$ \\
Control group & $83.93 \pm 7.13$ & $89.46 \pm 8.47$ & $125.60 \pm 20.00$ & $132.23 \pm 21.72$ \\
\hline
\end{tabular}

Notes: Compared to the control group, ${ }^{*} p<0.05$.

health-centered comprehensive nursing, provide the comprehensive nursing to the requirement of physiology, psychology and social aspect. Our investigation shows that the anxiety, fear, self-felling, muscular tension, blood pressure, pulse, intraoperative pain, postoperative pain, postoperative emotion, off-bed activity etc. during the perioperative period had obvious psychosocial factor. Therefore, it is appropriate and feasible to use psychological intervention. The psychological factor plays an important role during the occurrence, development and treatment of the disease. The poor psychological factor can result in disease and lead the symptom to aggravate. The psychological intervention is performed to the conscious patients during the perioperative period. The most important of all, must have a close relationship with the patient to be successful. The emotional rapport and high credibility is the key of successful intervention. The psychological intervention of perioperative patients could weaken or eliminate the psychological problems such as anxiety, fear, depression etc. Guide the patients to take suitable adaptive behavior, in order to control or relieve the various discomfort [3], could help the patients to spend the perioperative period safely. The psychological intervention could reduce obviously surgical complications and the sensibility to postoperative pain by adjusting the feeling of anxiety and fear to surgery and pain. In general, the time within three days after surgery is the critical period of pain, the effective psychological intervention could adjust the psychological environment of the patients, relieve the psychological burden, increase pain threshold. Provide positively active encouragement and care to the appropriately positive behavior, in order to correct the improper pain behavior [4]. Pain is defined by the International Association for the Study Pain as follow: "pain is an unpleasant sensory and emotional experience, the experience is related to the actual or potential damage, or pain is a kind of phenomenon that the patients' complaint from the point of hurt". Especially at the time of 8-36 h after surgery, even more should provide reasonable psychological intervention to the incision pain. Pain is not purely physical problems, various psychological factors and social factors could influence the occurrence and the evaluation of pain to some extent. Anxiety, tension and fear also could influence the pain threshold of the patients, so that the slight pain can cause severe reactions. The patients with open cholecystectomy would manifest depression, anxiety, fear and restlessness due to pain after surgery. The patients are extremely sensitive, they require not only technical treatment but more emotional care. Therefore, the psychological nursing appears more important. The psychological nursing effect score scale is prepared under the guidance of patient-centered nursing. The psychological nursing is performed by the score scale, it can make the nurses to focus on the purpose and the items of the psychological nursing as soon as possible, comprehensively understand the psychological condition of the patients quickly. According to the score requirement, the nurses not only inspect the general signs of patients, but also should learn to read people and their moods. In the course of the psychological nursing, the nurses should improve constantly self-quality and the language skills of psychological nursing, discern the psychological condition of patients, and provide the targeted psychological nursing. They should be sincere, friendly, gentle, euphemistic and considerate. The nurses can take part in the grade under the guide of the score scale, in order to measure the quality of nursing, plays the role of mutual supervision and impe- 
tus. In addition, it can reflect the achievement of operating room nurses during the psychological nursing work by the scoring method, overcome the wrong thinking that the result is the same with or without the psychological nursing, and enhance enthusiasm to work of the nurses. It can also reflect the quality and skills of psychological nursing of each nurse by the scoring method, stimulate their enthusiasm. To do a good job in psychological nursing, not only requires the nurse to have various knowledges of professional theory, psychology, education, ethic, philosophy and aesthetics etc., but also requires the nurse to learn new knowledge and accumulate experience constantly, improve constantly self-quality. By the effect scoring method, we can detect the deficiencies and flaws of psychological nursing and adjust the nursing plan timely, seek the reason of low score, correct the wrong timely and improve the quality of the psychological nursing. Practices show that when the perioperative psychological nursing is guided by the psychological nursing affects score scale, the quality of psychological nursing indeed undergone a qualitative change.

\section{Conflicts of interest}

These authors have no conflicts of interest to declare.

\section{Authors' contributions}

These authors contributed equally to this work.

\section{References}

1. Zhang SS. The Psychometric Properties and the Development of the Indicators of Quality Nursing Work Environments. Nursing Psychology. 2003;9(1):146-147.

2. Jiang QJ. Outcomes of Patients Discharged to Skilled Nursing Facilities After Acute Care Hospitalizations. Journal of Nursing Medicine. 2001;12(3):208-211.

3. Liu Y, Yang CQ. The role of psychological intervention in alleviating lumbar puncture fear and headache and osphyalgia. Journal of Nursing Science. 2000;15(12):707-709.

4. Dai XY. Exploratory Evidence of Schedule Control and Nursing Home Quality in Resident Care. Journal of Hospital Care. 2003;6(5):232-239. 